\title{
Application of the IMM-JPDA Filter to Multiple Target Tracking in Total Internal Reflection Fluorescence Microscopy Images
}

\author{
Seyed Hamid Rezatofighi ${ }^{1,2}$, Stephen Gould ${ }^{1}$, Richard Hartley ${ }^{1,3}$, \\ Katarina $\mathrm{Mele}^{2}$, and William E. Hughes ${ }^{4,5}$ \\ 1 College of Engineering \& Computer Sci., Australian National University, ACT, AU \\ 2 Quantitative Imaging Group, CSIRO Math., Informatics \& Statistics, NSW, AU \\ 3 National ICT (NICTA), AU \\ 4 The Garvan Institute of Medical Research, NSW, AU \\ 5 Department of Medicine, St. Vincent's Hospital, NSW, AU \\ hamid.rezatofighi@anu.edu. au
}

\begin{abstract}
We propose a multi-target tracking method using an Interacting Multiple Model Joint Probabilistic Data Association (IMMJPDA) filter for tracking vesicles in Total Internal Reflection Fluorescence Microscopy (TIRFM) sequences. We enhance the accuracy and reliability of the algorithm by tailoring an appropriate framework to this application. Evaluation of our algorithm is performed on both realistic synthetic data and real TIRFM data. Our results are compared against related methods and a commercial tracking software.
\end{abstract}

Keywords: Multi-Target tracking, Bayesian tracking, IMM Filter, Data association, JPDA filter, Total internal reflection fluorescence microscopy.

\section{Introduction}

Many biological mechanisms such as intracellular trafficking involve the interaction of diverse subcellular components moving between different intracellular locations and cellular membrane [1]. Analyzing these movements is an essential preliminary step in understanding many biological processes. This spatiotemporal analysis has become feasible using recent developments in time-lapse fluorescence imaging, such as total internal reflection fluorescence microscopy (TIRFM). However, manual scrutiny of hundreds of moving subcellular structures over numerous sequences is painstakingly slow and suffers from poor accuracy and repeatability. Therefore, the development of a reliable automated tracking algorithm is required for better biological exploration. However, many challenging difficulties such as high levels of noise, high object densities and intricate motion patterns confront the development of reliable automated tracking algorithms. Moreover, the subcellular structures generally have complex interactions while entering, exiting or temporarily disappearing from the frame [2].

Bayesian tracking approaches are a class of tracking algorithm that have became popular for cell tracking applications in recent years [2 8]. These tracking 
methods can properly deal with the interaction between the targets and long disappearance intervals by incorporating prior knowledge of object dynamics and measurement models. Particle filtering (PF) methods are a type of Bayesian tracking technique well suited to nonlinear models. Therefore, they have been applied in many biological applications [3, 5]. However, the main weakness of these methods is their high computation cost [3]. For this reason, Kalman filtering based methods, such as the interacting multiple model (IMM) filter, which are computationally effective, are still a popular alternative for biological applications [2, 4, 6]. In order to solve the measurement-to-target assignment problem, accurate multi-target tracking also requires robust data association. Some examples of these algorithms used in cell tracking applications include multiple hypothesis tracking (MHT) [7] and joint probabilistic data association (JPDA) [8]. MHT is a preferred technique for solving the data association problem due to considering all possible measurement-to-track assignments for a number of successive frames. However, it is computationally intensive, especially in the regions with high target density [9]. JPDA is a special case of MHT and considers all possible measurement-to-target assignments in each frame separately. In diverse applications, this data association technique provides acceptable performance whilst having significantly less processing time compared to the MHT algorithm. Moreover, it has been shown that for tracking highly maneuvering targets in the presence of clutter, the PDA-based filter in conjunction with the IMM filter yields one of the best solutions and has comparable performance to MHT [9].

Since in our application the objects of interest embody nonlinear dynamics we propose a combination of the IMM and the JPDA filters. The combination of these filters, the so called IMM-JPDA filter, was first introduced by BarShalom et al. 10] and has been used in various applications such as radar [1] and robotics [12]. However, to our knowledge, this is the first application of the IMM-JPDA filter to biological imaging. As the main contribution of this paper we tailor a framework for tracking vesicles in TIRFM images. We evaluate the performance of our algorithm on realistic synthetic sequences as well as a real TIRFM data set. In addition, our results are compared with those of related methods and one popular commercial software package. The results show that our algorithm is robust enough to track different vesicle dynamics whilst maintaining tracks after their temporary disappearance.

\section{Background}

In this section, we briefly review the IMM and JPDA filters. For a complete treatment see Blackman and Popoli [13]. The IMM filter is a Bayesian state estimation algorithm which models nonlinear dynamic and measurement equations using multiple switching linear models. For each model $k$, the posterior density $p\left(\mathrm{x}_{t} \mid \mathrm{Z}_{t}, k_{t}=k\right)$, where $\mathrm{x}_{t}$ is the system state vector and $\mathrm{z}_{t}$ is the measurement vector at time $t$, evolves based on the Kalman filter equations [13]. The switching between models is regulated by a transition probability matrix. To this end, IMM switching weights, so called IMM model probabilities $\lambda_{t}^{k}$, are calculated 
and updated at each time index $t$ using this matrix. Next, these weights are used for mixing the posterior densities of each model $p\left(\mathrm{x}_{t} \mid \mathrm{z}_{t}, k_{t}\right)$ and calculating the final posterior density $p\left(\mathrm{x}_{t} \mid \mathrm{z}_{t}\right)$.

The JPDA filter is a method of associating the detected measurements in the current frame with existing targets using a joint probabilistic score. This score is calculated based on the set of all valid joint associations, $\Theta_{j i}$, which assign measurement $j$ to target $i$. Here, a set of all possible measurement-to-track hypotheses are first generated such that each detected measurement is uniquely chosen by one track in each hypothesis. A null assignment $\emptyset$, representing the assignment of no observation to a given track, is also considered. Next, the probability $P\left(\theta \mid z_{t}\right)$ corresponding to each hypothesis, $\theta$, is calculated. In the case of linear Gaussian models, this probability can be calculated as $P\left(\theta \mid \mathrm{z}_{t}\right) \propto$ $\prod_{(i, j) \in \theta} g_{i j}$, where $g_{i j}$ is a likelihood function,

$$
g_{i j}= \begin{cases}1-P(D) & \text { if } j=\emptyset \\ \frac{\exp \left(-d_{i j}^{2} / 2\right)}{(2 \pi)^{M / 2} \sqrt{|S|}} & \text { otherwise }\end{cases}
$$

where $P(D)$ is the probability of detection, $M$ is dimension of the measurement, and $S$ is innovation covariance matrix of the Kalman filter. Here $d_{i j}$ is the normalized statistical distance between track $i$ and measurement $j$ using the innovation covariance matrix $S$.

Consequently, the joint probabilistic score, $\beta_{j i}$, that measurement $j$ was generated by track $i$ is obtained by $\sum_{\theta \in \Theta_{j i}} P\left(\theta \mid \mathrm{z}_{t}\right)$. Finally, tracks are updated with a weighted sum of measurements where the weights are the score probabilities.

\section{Method}

An accurate multiple target tracking algorithm requires robust detection for track initialization, initiation, and termination. To this end, we use the maximum possible h-dome (MPHD) method [14]. This detection method is accurate enough to detect most of targets with a low false detection rate. The output of the algorithm is a set of detected positions and an estimated background $\mathbf{B}_{t}$ in frame $t$ (see Rezatofighi et al. [14] for details). Then, we use an enhanced IMM-JPDA filter along with a track management procedure as follows.

\subsection{An Enhanced IMM-JPDA Filter}

Since the data in our case consists of two dimensional sequences, the state vector and the measurement vector are typically defined as $\mathrm{x}_{t}=\left(x_{t}, \dot{x}_{t}, y_{t}, \dot{y}_{t}\right)$, including positions $\dot{\mathrm{x}}_{t}=\left(x_{t}, y_{t}\right)$ and velocities $\dot{\mathrm{x}}_{t}=\left(\dot{x}_{t}, \dot{y}_{t}\right)$, and $\mathrm{z}_{t}=\left(\hat{x}_{t}, \hat{y}_{t}\right)$ respectively. In the IMM-JPDA filter, the prediction density of the state $\mathrm{x}_{t} \in \mathbb{R}^{4}$ in each model is first estimated based on each linear dynamic model of the IMM filter. Next, each density is updated by the JPDA filter. Last, the posterior density $p\left(\mathrm{x}_{t} \mid \mathrm{Z}_{t}\right)$ is calculated based on a weighted combination of posterior densities $p\left(\mathrm{x}_{t} \mid \mathrm{Z}_{t}, k_{t}\right)$ using the IMM model probabilities. To improve the performance of this filter in TIRFM sequences, we tailor the framework as described below. 
IMM with a State Dependent Transition Probability Matrix. To deal with nonlinear dynamics of the biological structures, different numbers of linear dynamic models have been proposed in the literature [3, 4, 6]. Although, a large number of linear dynamics [4, 6] may result a better estimation of these nonlinear dynamics, they are more computationally demanding. In addition, more dynamic models increase the uncertainty of the estimated state; because in the IMM filter, a mixture of weighted Gaussian posterior densities results in higher variance. For these reasons, we use the two dynamic models defined by Smal et al. 3] including random walk and nearly constant velocity motion with small accelerations. These two types of dynamics properly model the nonlinear motion of the vesicles in TIRFM sequences. The random walk and constant velocity models resemble vesicle motion patterns described as tethering and docking, and linear movements, respectively [1]. Also for abrupt changes in direction, the random walk model operates as the transition state between two linear movements.

Traditionally, the elements of the transition probability matrix (TPM) are almost always considered as constant and chosen empirically. In Li et al. [4], these values are adaptively improved using an online minimum mean-square error estimation. However, it is still a shared matrix for all targets and requires an assumed distribution for probabilities of TPM. In contrast, we use an adaptive transition probability matrix which evolves based on the state of each target in the previous frame. Biologically, a vesicle can occasionally switch between these two states based on its kinetic energy. In other words, a vesicle with low velocity is more likely to either remain in the first model (docking and tethering dynamics) $\left(k_{t}=k_{t-1}=1\right)$ or switch from the linear movement model $\left(k_{t-1}=2\right)$ to the first model. The transition probability for the above states can be modeled by a decreasing function of the velocity of each target in the previous frame $\dot{\mathrm{X}}_{t-1}$. Here, we define this function by a Gaussian-like probability function as

$$
P\left(k_{t} \mid k_{t-1}=k, \dot{\mathrm{x}}_{t-1}\right)= \begin{cases}\mathcal{S}^{k} \exp \left(-\frac{1}{2} \dot{\mathrm{x}}_{t-1}\left(\mathcal{A}^{k}\right)^{-1} \dot{\mathrm{x}}_{t-1}\right), & k_{t}=1, \\ 1-\mathcal{S}^{k} \exp \left(-\frac{1}{2} \dot{\mathrm{x}}_{t-1}\left(\mathcal{A}^{k}\right)^{-1} \dot{\mathrm{x}}_{t-1}\right), & k_{t}=2,\end{cases}
$$

where $\mathcal{S}^{k}$ is the maximum switching probability from model $k_{t-1}$ to model $k_{t}$ and $\mathcal{A}^{k}$ is a user specified positive semi-definite matrix. In our work, we set $\mathcal{A}^{k}=\sigma^{k} \mathbf{I}$, where $\mathbf{I}$ is the identity matrix. These parameters are fixed for each model based on prior knowledge. Finally, the elements of the transition probability matrix can be obtained by marginalization over $\dot{\mathrm{x}}_{t-1}$ :

$$
P\left(k_{t} \mid k_{t-1}, \mathrm{z}_{t-1}\right)=\int P\left(k_{t} \mid k_{t-1}, \dot{\mathrm{x}}_{t-1}\right) p\left(\dot{\mathrm{x}}_{t-1} \mid \mathrm{z}_{t-1}, k_{t-1}\right) d_{\dot{\mathrm{x}}_{t-1}} .
$$

Since $p\left(\dot{\mathrm{X}}_{t-1} \mid \mathrm{Z}_{t-1}, k_{t-1}=k\right)$ is a Gaussian with mean $\dot{\mu}_{t-1}^{k}$ and covariance $\dot{\Sigma}_{t-1}^{k}$, $P\left(k_{t} \mid k_{t-1}=k, \mathrm{z}_{t-1}\right)$ can be written in a closed form as

$$
\mathcal{S}^{k} \sqrt{\frac{\left|\mathcal{A}^{k}\right|}{\left|\dot{\Sigma}_{t-1}^{k}+\mathcal{A}^{k}\right|}} \exp \left(-\frac{1}{2}\left(\dot{\mu}_{t-1}^{k}\right)^{T}\left(\dot{\Sigma}_{t-1}^{k}+\mathcal{A}^{k}\right)^{-1}\left(\dot{\mu}_{t-1}^{k}\right)\right), \quad \text { for } k_{t}=1 .
$$

For the case $k_{t}=2$, this probability is simply one minus the above. 
An Enhanced JPDA for Vesicle Tracking. The performance of the JPDA algorithm is enhanced if the probability of detection in Equation 1 is a function of the position of each target. The detection of each target directly depends on the performance of the detection algorithm. In MPHD, a missed detection is more likely to occur at the edge of background structures. Therefore, the distribution of this probability can be modeled as a function of the background $\mathbf{B}_{t}$ estimated by the MPHD method. In this paper, we define this probability as $P\left(D \mid \hat{\mathrm{x}}_{t \mid t-1}^{k}\right)=$ $1-\Omega\left(\left\|\nabla \mathbf{B}_{t}(\dot{\mathrm{x}})\right\|\right)_{\dot{\mathrm{x}}=\dot{\mathrm{x}}_{t \mid t-1}^{k}}$, where $\|\nabla \cdot\|$ is the gradient magnitude operator and $\Omega(\cdot)$ is a function that normalizes its argument to the interval $[0,1]$. Because a closed form for the probability of detection by marginalization over $x_{t \mid t-1}^{k}$ can not be calculated, we approximate it by the point estimate $P\left(D \mid \mu_{t \mid t-1}^{k}\right)$.

In the 2D TIRFM imaging system, the emitting intensity of each fluorescence object is a nonlinear function of its depth as $z_{t}-z_{0}=\zeta \log \left(I_{0} / I_{t}\right)$, where $\zeta$ is a decaying factor and $z_{0}$ and $I_{0}$ are a known depth and its equivalent intensity, respectively. Therefore, we can use the relative changes in the target's depth movement to better assign each measurement to its corresponding targets. To this end, we assume that the maximum intensity $\left(I_{\max }\right)$ corresponds to $z_{0}=0$. By extending the state vector to $\mathrm{x}_{t}=\left(x_{t}, \dot{x}_{t}, y_{t}, \dot{y}_{t}, z_{t}, \dot{z}_{t}\right)$ and the measurement vector to $\mathrm{z}_{t}=\left(\hat{x}_{t}, \hat{y}_{t}, \hat{z}_{t}=\zeta \log \left(I_{\max } / I_{t}\right)\right)$, the performance of the JPDA algorithm is enhanced.

\subsection{Track Management}

Initialization and Track Initiation. In order to initialize the trajectories, we apply the MPHD method for the first two consecutive sequences. The positions are initialized with the output of this method for the first frame. The initial velocities are estimated as the difference between the detected positions in the first frame and the nearest corresponding detected positions in the second frame. For track initiation of newly appearing targets, we use the joint probabilistic score described in $₫ 2$. To this end, a total joint probabilistic score $\beta_{j i}^{\mathcal{K}}$ is first calculated as $\sum_{k=1}^{2} \lambda_{t}^{k} \beta_{j i}^{k}$, where $\beta_{j i}^{k}$ is the joint probabilistic score of each model and $\lambda_{t}^{k}$ are the IMM weights. Next, the detected measurements in frame $t$ with the highest total score are considered as the most plausible measurements for the existing targets in this frame. The remaining detected measurements are initiated as new born targets.

Temporary Target Disappearance and Track Termination. Due to the depth movements of the targets in TIRFM sequences, they can temporarily disappear for several sequential frames. In this situation, the first element of the total joint probabilistic score $\left(\beta_{\emptyset i}^{\mathcal{K}}\right)$ representing the missed detection probability is maximum in the frames where a target has either disappeared or was not detected. To deal with this temporary disappearance, our algorithm is allowed to continue tracking missed detections for up to $N$ consecutive frames. Otherwise, the track is terminated from the last frame where the maximum score is not allotted to the $\beta_{\emptyset i}^{\mathcal{K}}$. 


\section{Experimental Results}

The proposed tracking algorithm was evaluated using synthetic data (with ground truth) and also real TIRFM sequences. To show the efficacy of the combination of IMM and JPDA filters, we compared the performance of the proposed method (IMM-JPDA) with those of two related methods including the JPDA filter with a small acceleration dynamic model [8] and IMM filter along with a data association technique using the innovation matrix [6]. We also validate our experiments against a state-of-the-art commercial tracking software package (ImarisTrack).

In the first experiment, the tracking methods were tested using realistic synthetic sequences. The synthetic data consists of 80 targets moving through 40 frames inside a $450 \times 450$ pixel region. The spatial intensity profile of the targets were modeled by a $2 \mathrm{D}$ Gaussian distribution and were generated in different sizes similar to the size of the vesicles in the real TIRFM. Furthermore, due to the 3 -D dimensional motion of the targets, their intensity is modulated according to their depth (see 33.1 ). To add an appropriate background, the background of a real TIRFM image estimated using the MPHD method [14], was added to the generated synthetic sequences. Next, the sequences were contaminated with Poisson noise. The dynamics of the targets were modeled using two aforementioned models. Also, targets can switch between these two dynamics (Fig. 1).

In order to quantitatively assess the performance of the tracking methods, we need an appropriate measure to characterize different aspects of tracking performance such as track accuracy, track truncation, data association and missed or false tracks. Metrics used in previous works [2 8] can not properly represent the performance of a multi-target tracking algorithm. Recently, a metric based on optimal subpattern assignment (OSPA) has been introduced by Risitc et al. [15] that captures the aforementioned aspects by a single value. This value can be seen as the sum of two errors including cardinality and location errors. The cardinality error can be interpreted as errors related to missed or false tracks while location error shows track accuracy error and labeling error. In other words, the accuracy of a tracking filter in tracking of a target and the performance of its data association technique are better shown by location error. On the other hand, a truncated track increases both cardinality and location errors because of missed tracks in the gaps and labeling error of truncated tracks.

In Table 1, the performance of the IMM, JPDA, IMM-JPDA and ImarisTrack for realistic synthetic data is compared using OSPA metric. For the first three methods, same detection scheme (MPHD) was used. As a result, they track similar false targets and have similar cardinality error. Differences in this error are due to their performance in filling the gaps between two tracks (missed track error). This error is noticeably higher for the ImarisTrack software because of its different detection scheme. The location error in this table demonstrates the performance of both the tracking filter and data association technique. As expected, selection of the JPDA filter as data association technique enhances the performance of the tracking system. However, the JPDA filter can not track nonlinear dynamics as well as the IMM-JPDA filter. Fig. 1(b)-(e) shows results from the IMM-JPDA method for some complex situations. 
Table 1. Comparison of the performance of the IMM, JPDA, IMM-JPDA and ImarisTrack for realistic synthetic data using OSPA metric (lower value is better)

\begin{tabular}{|c||c|c|c|c|}
\hline Methods & IMM [6] & JPDA [8] & IMM-JPDA & ImarisTrack \\
\hline \hline OSPA [15] & 7.81 & 5.78 & $\mathbf{5 . 4 5}$ & 13.61 \\
\hline Cardinality error & 2.68 & 2.65 & $\mathbf{2 . 3 6}$ & 6.75 \\
\hline Location error & 5.13 & 3.13 & $\mathbf{3 . 0 9}$ & 6.86 \\
\hline
\end{tabular}
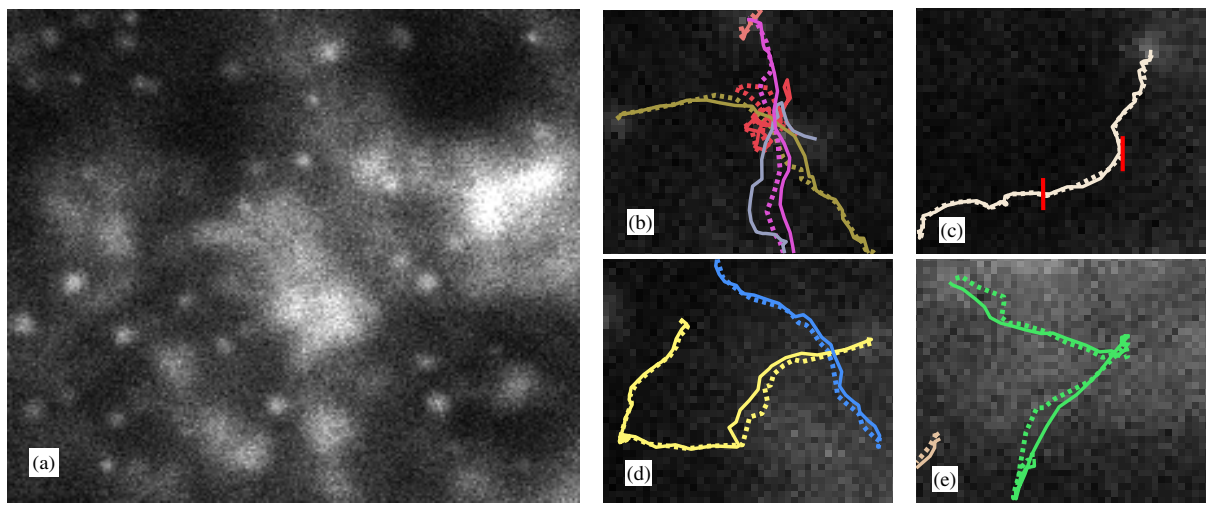

Fig. 1. (a) A part of the realistic synthetic data with locally varying $\mathrm{SNR}=2-6$. (b)(e) The result of tracking using the proposed algorithm (dashed line) and the ground truth (solid line): (b) a complex assignment, (c) a temporary disappearance (between two red lines), (d) a maneuvering motion, and (e) a switching dynamics

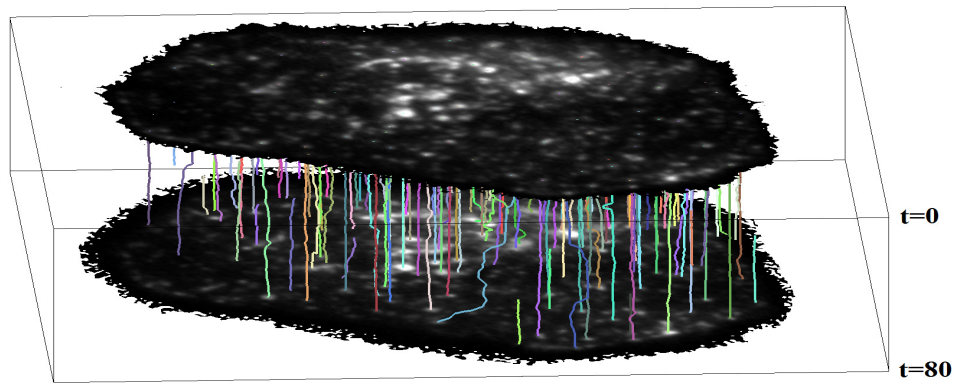

Fig. 2. Tracking result of the proposed method for 80 real TIRFM sequences

The described tracking methods were also tested on real TIRFM sequences (Fig. 2). Since the ground truth was not available for these real data, the results of the tracking were only visually evaluated by expert biologist. Our method in many cases such as temporary disappearance of the targets and their maneuvering or switching dynamics outperforms the other methods. 


\section{Conclusion}

Due to complex interaction of subcellular structures, the performance of tracking systems can be noticeably affected by their method for data association. Our results show that the combination of IMM with JPDA can be effective in both tracking nonlinear dynamics and solving the complex measurement-to-track assignment problem. Moreover, our method has significantly lower processing time with comparable performance to particle filter based approaches. Specifically, it takes only few minutes to track hundreds of targets using our method. This suggests that our method can be used as a reliable algorithm for tracking hundreds moving targets in long TIRFM sequences.

\section{References}

1. Burchfield, J., Lopez, J., Mele, K., Vallotton, P., Hughes, W.: Exocytotic vesicle behaviour assessed by TIRFM. Traffic 11, 429-439 (2010)

2. Feng, L., Xu, Y., Yang, Y., Zheng, X.: Multi. Dense particle tracking in fluoresc. microsc. images based on MAP. J. Struct. Biol. 173(2), 219-228 (2011)

3. Smal, I., Meijering, E., Draegestein, K., Galjart, N., Grigoriev, I., Akhmanova, A., Van Royen, M., Houtsmuller, A., Niessen, W.: Multi. object tracking in molecular bioimag. by rao-blackwellized marginal PF. Med. Image Anal. 12(6), 764-777 (2008)

4. Li, K., Miller, E., Chen, M., Kanade, T., Weiss, L., Campbell, P.: Cell population tracking and lineage construction with spatiotemporal context. Med. Image Anal. 12(5), 546-566 (2008)

5. Smal, I., Draegestein, K., Galjart, N., Niessen, W., Meijering, E.: PF for multi. object tracking in dynamic fluoresc. microsc. images: Application to microtubule growth analysis. IEEE Trans. Med. Imag. 27(6), 789-804 (2008)

6. Genovesio, A., Liedl, T., Emiliani, V., Parak, W., Coppey-Moisan, M., Olivo-Marin, J.: Multi. particle tracking in 3-D+t microsc.: Method and application to the tracking of endocytosed quantum dots. IEEE Trans. Image Process., 1062-1070 (2006)

7. Chenouard, N., Bloch, I., Olivo-Marin, J.: MHT in microsc. images. In: ISBI 2009, pp. 1346-1349 (2009)

8. Smal, I., Niessen, W., Meijering, E.: A new detection scheme for multi. object tracking in fluoresc. microsc. by JPDA filtering. In: ISBI 2008, pp. 264-267 (2008)

9. Bar-Shalom, Y., Kirubarajan, T., Lin, X.: PDA techniques for target tracking with applications to sonar, radar and EO sensors. IEEE Aerosp. Electron. Syst. Mag. 20(8), 37-56 (2005)

10. Bar-Shalom, Y., Chang, K.C., Blom, H.A.P.: Tracking splitting targets in clutter using an IMM JPDA filter. In: CDC 1991, pp. 2043-2048 (1991)

11. Chen, B., Tugnait, J.K.: Tracking of multi. maneuvering targets in clutter using IMM/JPDA filtering and fixed-lag smoothing. Automatica 37(2), 239-249 (2001)

12. Hoffmann, C., Dang, T.: Cheap joint probabilistic data association filters in an interacting multiple model design. Robot. Auton. Syst. 57(3), 268-278 (2009)

13. Blackman, S., Popoli, R.: Design and analysis of modern tracking systems, vol. 685. Artech House, Norwood (1999)

14. Rezatofighi, S.H., Hartley, R., Hughes, W.E.: A new approach for spot detection in TIRFM. In: ISBI 2012, pp. 860-863 (2012)

15. Ristic, B., Vo, B., Clark, D., Vo, B.: A metric for performance evaluation of multitarget tracking algorithms. IEEE Trans. Signal Process. 59(7), 3452-3457 (2011) 